INPLASY

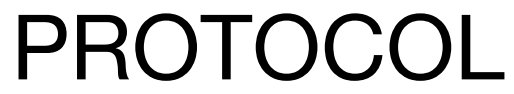

To cite: Qiu et al. Amputation risk with canagliflozin, empagliflozin, and dapagliflozin: a network metaanalysis of large randomized trials and cohort studies. Inplasy protocol 202090071. doi:

10.37766/inplasy2020.9.0071

Received: 18 September 2020

Published: 18 September 2020

Corresponding author:

Mei Qiu

3798214835@sina.cn

Author Affiliation:

Shenzhen Longhua District

Central Hospital

Support: None.

Review Stage at time of this submission: Preliminary searches.

Conflicts of interest:

None.

\section{Amputation risk with canagliflozin, empagliflozin, and dapagliflozin: a network meta-analysis of large randomized trials and cohort studies}

Qiu, M1; Ding, LL2; Zhang, M3; Lin, JH; Zhou, HR5.

Review question / Objective: Whether various sodium-glucose cotransporter-2 inhibitors (SGLT2is) assessed in large cardiovascular outcome trials increase the risk of amputation remains controversial.

Condition being studied: This network meta-analysis will assess the relative risk of different SGLT2is versus nonSGLT2i antihyperglycemic drugs or placebo in leading to amputation.

Information sources: We will systematically search PubMed and Embase using pre-defined search strategies, for relevant large sample studies.

INPLASY registration number: This protocol was registered with the International Platform of Registered Systematic Review and Meta-Analysis Protocols (INPLASY) on 18 September 2020 and was last updated on 18 September 2020 (registration number INPLASY202090071).

\section{INTRODUCTION}

Review question / Objective: Whether various sodium-glucose cotransporter-2 inhibitors (SGLT2is) assessed in large cardiovascular outcome trials increase the risk of amputation remains controversial.
Condition being studied: This network meta-analysis will assess the relative risk of different SGLT2is versus non-SGLT2i antihyperglycemic drugs or placebo in leading to amputation. 


\section{METHODS}

Search strategy: PubMed Search: (canagliflozin[MH] OR canagliflozin[TIAB] OR Invokana[TIAB] OR empagliflozin [Supplementary Concept] OR empagliflozin[TIAB] OR Jardiance[TIAB] OR “ 2 - ( 3 - ( 4 - e th oxy benzyl) - 4 chlorophenyl)-6-hydroxymethyltetrahydro -2H-pyran-3,4,5-triol"[Supplementary Concept] OR dapagliflozin[TIAB] OR forxiga[TIAB] OR Sodium-Glucose Transporter 2 Inhibitors[MH] OR "sodiumglucose cotransporter-2 inhibitors"[tiab] OR "sodium-glucose cotransporter-2 inhibitor"[tiab] OR "sodium-glucose transporter-2 inhibitors"[tiab] OR "sodiumglucose transporter-2 inhibitor"[tiab] OR "SGLT-2 Inhibitors"[TIAB] OR "SGLT2 Inhibitors"[TIAB] OR "SGLT-2 Inhibitor"[TIAB] OR "SGLT2 Inhibitor"[TIAB] OR "SGLT2i"[TIAB] OR "SGLT2is"[TIAB] OR "SGLT-2i"[TIAB] OR "SGLT2-is"[TIAB]) AND (Amputation[MH] OR Amputation[TIAB]). Embase Search: (canagliflozin/exp OR canagliflozin:ab,ti OR Invokana:ab,ti OR empagliflozin/exp OR empagliflozin:ab,ti OR dapagliflozin/exp OR dapagliflozin:ab,ti OR 'sodium glucose cotransporter 2 inhibitor'/exp OR 'sodium-glucose cotransporter-2 inhibitors':ab,ti OR 'sodium-glucose cotransporter-2 inhibitor':ab,ti OR 'sodium-glucose transporter-2 inhibitors':ab,ti OR 'sodiumglucose transporter-2 inhibitor':ab,ti OR 'SGLT-2 Inhibitors':ab,ti OR 'SGLT2 Inhibitors':ab,ti OR 'SGLT-2 Inhibitor':ab,ti OR 'SGLT2 Inhibitor':ab,ti OR 'SGLT2i':ab,ti OR 'SGLT2is':ab,ti OR 'SGLT-2i':ab,ti OR 'SGLT2-is':ab,ti) AND (Amputation/exp OR Amputation:ab,ti).

Participant or population: Adults with type 2 diabetes or chronic heart failure.

Intervention: Three SGLT2is (i.e., empagliflozin, canagliflozin and dapagliflozin). We will not consider doses of drugs as effect modifiers.

Comparator: Non-SGLT2i antihyperglycemic drugs or placebo.
Study designs to be included: Randomized controlled trial (RCT) and cohort study.

Eligibility criteria: They are detailed in the above PICOS sections.

Information sources: We will systematically search PubMed and Embase using predefined search strategies, for relevant large sample studies.

Main outcome(s): Amputation. At the end of follow-up, using hazard ratios (HRs) and 95\% confidence intervals (Cls) as reported by included studies.

Quality assessment / Risk of bias analysis: Two authors will independently use the Cochrane risk of bias assessment tool to assess risk of bias for included RCTs, and use the Newcastle-Ottawa Scale (NOS) to assess risk of bias for included cohort studies. Any disagreements related to risk of bias assessment will be resolved through discussion with a third author.

Strategy of data synthesis: We will use the study-level survival data (i.e., HRs and 95\% Cls extracted from included studies) perform random-effects conventional meta-analysis and network meta-analysis within a Bayesian framework to synthesize the estimators of relative effects. I square statistic will be calculated to measure statistical heterogeneity. The inconsistency plot will be drawn to assess the inconsistency between direct and indirect evidences when there are one or more closed loops observed in the evidence network plot. Comparison-adjusted funnel plots will be plotted to assess dominant publication bias. Network meta-analysis will be conducted in the $R$ (version 3.6.0) and JAGS (version 4.3.0) software. Other statistical analysis and statistical plots will be done in the Stata software (version 15.1).

Subgroup analysis: Not pre-planned.

Sensibility analysis: We will conduct sensitivity analysis by doing another network meta-analysis only based on the studies of type 2 diabetes. Meta-regression 
analysis will be conducted to evaluate the impact of study type and follow-up duration on the amputation risk with SGLT2is.

Country(ies) involved: China.

Keywords: canagliflozin, empagliflozin, dapagliflozin, SGLT2is, amputation.

Contributions of each author:

Author 1 - Mei Qiu.

Author 2 - Liang-Liang Ding.

Author 3 - Miao Zhang.

Author 4 - Jin-Hao Lin.

Author 5 - Hai-Rong Zhou. 